\title{
Estrategias para fortalecer el proceso de gestión del talento humano en una entidad pública*
}

\author{
Strategies to strengthen the human talent management \\ process in a public entity
}

\section{Estratégias para o fortalecimento do processo de gestão do talento humano em uma entidade pública}

Recibido: 10 de diciembre de 2018

Leidy Marcela Romero Parra**

Consultora independiente, Colombia

Mireys Salcedo Lara***

Consultora independiente, Colombia

Cómo citar: Romero Parra, L.M. y Salcedo Lara, M. (2019). Estrategias para fortalecer el proceso de gestión del talento humano en una entidad pública. Signos. Investigación en Sistemas de Gestión, 11(2), 99-117. Doi: https://doi.org/10.15332/24631140.5084

\section{RESUMEN}

Coralina es una entidad pública de San Andrés dedicada al medio ambiente, que cuenta con un sistema de gestión de la calidad. Esta entidad tiene un problema de pérdida de capital intelectual que se evidencia en las auditorías internas con el incumplimiento de algunas actividades, lo que afecta la calidad del servicio. En este sentido, la investigación tuvo como objetivo proponer estrategias para el fortalecimiento del proceso de gestión del talento humano en dicho sistema de gestión de calidad. Para ello se realizó una investigación cualitativa y se consideró el análisis de contenido de la información oral y escrita proporcionada por los funcionarios de la corporación. El diagnóstico arrojó

* Artículo de investigación.

** Magíster en Calidad y Gestión Integral, profesional en Salud Ocupacional. Línea de investigación en Gestión Pública, Convenio Universidad Santo Tomás-Icontec. Villavicencio, Colombia. Correo electrónico: leidyromerop@usantotomas.edu.co.

**** Magíster en Calidad y Gestión Integral, bacterióloga. Convenio Universidad Santo Tomás-Icontec. San Andrés, Colombia. Correo electrónico: mireyssalcedo@santotomas.edu.co. Orcid: https://orcid.org/0000-0003-1515-8049 
como resultado que hacen falta recursos para la buena gestión del talento humano y mayor compromiso, motivación y formación por competencias del personal, y que hay fallas en todos los componentes del proceso. Las estrategias propuestas para superar tales carencias o fallas, con su respectivo plan de acción, se enfocaron en cada uno de esos componentes, para articular el proceso con las normas de calidad relevantes, especialmente la ISO 9004:2009, que se orienta al éxito sostenido de las organizaciones con un enfoque de gestión de la calidad.

Palabras clave: capital humano, recursos humanos, desarrollo humano, información y calidad del producto, normalización y compatibilidad.

\section{ABSTRACT}

CORALINA is a public entity engaged in the environment sector, based on San Andrés, which has a quality management system in place. This entity has a problem elated to the loss of intellectual capital, which is evidenced in the internal audits by the noncompliance with some activities thus affecting service quality. In this regard, the research was aimed at proposing strategies for the strengthening of the human talent management process in the quality management system. A qualitative research was developed, and the content of written and oral information provided by the corporation officials was analyzed. The diagnosis revealed the lack of resources for a proper human talent management, increased commitment, motivation and competence-based training of the personnel, as well as faults in all the components of the process; therefore, the strategies proposed with their respective action plan were focused on all those components in order to articulate the process with quality standards, especially ISO 9004:2009 that is oriented toward the sustained success of organizations with a quality management approach.
Keywords: Human capital, human resources, human development, information and product quality, standardization and compatibility.

\section{RESUMO}

A CORALINA é uma entidade pública do arquipélago de San Andrés voltada ao meio ambiente, que possui um sistema de gestão da qualidade. Esta entidade apresenta uma problemática associada à perda de capital intelectual, o que se revela nas auditorias internas com a inadimplência de algumas atividades que afetam a qualidade do serviço. Nesta sequência, a pesquisa teve 0 objetivo de propor estratégias para o fortalecimento do processo de gestão do talento humano no sistema de gestão de qualidade. Para isso, foi realizada uma pesquisa qualitativa e considerou-se a análise de conteúdo das informações orais e escritas fornecidas pelos funcionários da corporação. 0 diagnóstico deu como resultado que ainda se precisa de recursos para a boa gestão do talento humano, maior engajamento, motivação e formação por competências do pessoal, bem como falhas em todos os componentes do processo, pelo que as estratégias colocadas com 0 seu plano de ação correspondente se focaram em todos esses componentes, para vincular o processo com as normas de qualidade, em particular a ISO 9004:2009 voltada ao sucessos sustentável das organizações com foco na gestão da qualidade.

Palavras-chave: Capital humano, recursos humanos, desenvolvimento humano, informação e qualidade do produto, normalização e compatibilidade. 


\section{INTRODUCCIÓN}

Hasta finales de la década de los ochenta el proceso de GTH (gestión del talento humano) no tenía mayor relevancia dentro de las organizaciones. Era una actividad básica e incipiente pues la baja rotación de personal, la lenta evolución de la tecnología y la estabilidad del mercado hacían que el proceso fuera sencillo: bastaba con encontrar una persona que reuniera las habilidades básicas para el cargo, cuyo proceso de formación consistía en un entrenamiento centrado en las funciones que desempeñaría y que podría ser promocionada más adelante, según su antigüedad en la empresa o el buen desempeño que exhibiera (Pereda y Berrocal, 2011).

Actualmente la situación es muy diferente. La GTH ha adquirido gran preponderancia en las organizaciones, las cuales han entendido que el TH (talento humano) es un factor de competitividad y diferenciación difícil de ser replicado por la competencia (Alles, 2006; 2016). En este sentido, el caso de Toyota es emblemático pues parte de la ventaja acumulada durante años ha estado fundamentada en el TH y su capacidad para "resolver problemas, innovar y constituirse en agente del cambio" (Hamel y Breen, 2012, p. 37).

En los SGC (sistemas de gestión de la calidad) el TH también resulta determinante para asegurar el correcto desempeño y la eficacia del sistema. Por eso es imprescindible fortalecer sus competencias (toma de conciencia, conocimiento, compromiso, liderazgo, entre otros aspectos), que son fundamentales para el cumplimiento de los principios de calidad e inciden en la satisfacción del cliente. De tal manera que tanto el compromiso de la alta dirección como la adhesión del TH son presupuestos básicos para el éxito de cualquier SGC (Sánchez, 2011). Máxime cuando la calidad es un concepto multidimensional que en la mayoría de sus dimensiones involucra de alguna manera al TH (Petrick y Furr, 2003; Atehortúa,
Bustamante y Valencia, 2008; Gutiérrez y Rubio, 2009; Benavides y Quintana, 2015; entre otros).

La relación entre GTH y SGC es de doble vía, y la implementación de un SGG impacta también al TH. Un estudio llevado a cabo en una institución educativa de la ciudad de Pasto, a fin de conocer los efectos de la implementación del SGC bajo el modelo NTC-ISO 9001 en la gestión del recurso humano y a partir de la definición de siete categorías (gestión directiva, participación del personal, gestión documental, ambiente de trabajo, desempeño del personal basado en competencias, comunicación y, mejora continua), plantea que, como resultado de la implementación, la formación y capacitación del personal ha tenido una notable influencia en otras categorías (gestión directiva, gestión documental, ambiente de trabajo y mejora continua), lo que ha potenciado habilidades de liderazgo y trabajo en equipo y ha contribuido a mejorar el desempeño del personal y sus competencias laborales (Estacio Maigual, 2012).

En otro estudio similar al anterior, orientado a determinar los efectos de la implementación del SGC en la gestión humana del Centro de Gestión Administrativa del Sena, los autores llegan más o menos a las mismas conclusiones: la mejora continua ha fortalecido las competencias del TH que trabaja para la institución y el liderazgo ejercido por la alta dirección de la entidad ha contribuido a la eficacia del sistema, lo cual se ve reflejado en la fluidez de la comunicación y la información (Méndez y Fandiño, 2010).

Lo anterior pone de presente que lograr la colaboración y adhesión del factor humano en los SGC demanda un esfuerzo adicional. La mera transmisión de información es insuficiente pues también se requiere de un ambiente de trabajo adecuado. Así se desprende de otra investigación en la cual participaron doce empresas, la mayoría de ellas multinacionales, cuyos autores concluyen que la mera introducción de un sistema de información eficiente no 
garantiza la transferencia y creación de conocimiento: es necesario un clima organizacional propicio que debe tener como características la confianza mutua, el acceso a la ayuda, la empatía activa y el juicio clemente, en los equipos de trabajo (Zárraga y Bonache, 2005).

En el caso de la entidad pública objeto del presente estudio, cuenta con un sistema integrado de gestión que toma como referencias el Meci (Modelo Estándar de Control Interno) 1000:2005, el Sisteda (Sistema de Desarrollo Administrativo) 2005 y el Sistema de Gestión de Calidad bajo NTCGP 1000:2009 e ISO 9001:2008 con enfoque basado en procesos. Eso le ha permitido gestionar sus objetivos de acuerdo a su misión, visión, PGAR (Plan de Gestión Ambiental Regional), PA (Plan de Acción) y sus políticas y proyectos.

Ahora bien, aunque la entidad lleva a cabo un proceso de selección del personal con diferentes filtros para asegurar la idoneidad del TH contratado, se ha podido observar que después de implementado el SGC paulatinamente se han perdido el conocimiento, el compromiso y la motivación del TH. El resultado se refleja en los informes de las auditorías internas, donde se evidencia la omisión de algunas actividades que demanda el SGC.

Situación problemática si se tiene en cuenta que los SGC son una herramienta de gran importancia estratégica, tal y como están definidos en la NTC ISO 9001:2008: "conjunto de elementos mutuamente relacionados o que interactúan para establecer la política y los objetivos, para dirigir y controlar una organización con respecto a la calidad" (Icontec, 2005, p. 11). Cabe resaltar el numeral 6.2 de dicha norma, que establece la competencia del TH fundamentada en la educación, la formación, las habilidades y la experiencia apropiadas. Téngase en cuenta además la existencia de un principio de calidad especialmete importante: el de participación del personal a todos los niveles, el cual permite que las habilidades del $\mathrm{TH}$ sean usadas en beneficio de la organización.
Dicha problemática refleja además que el personal no se encuentra inmerso ni participa activamente en la mejora continua del SGC implementado. Simultáneamente, demuestra desmotivación y falta de compromiso, da la impresión de que trabajan solamente para dar cumplimiento a las actividades y entregar los productos contratados dejando de lado otros aspectos igual de importantes, como el empoderamiento y la realización de las actividades del SGC.

Por otra parte, algunos funcionarios se retiran debido a que acceden a empleos con mejores garantís y beneficios laborales, y se llevan consigo el conocimiento, que muchas veces no se encuentra documentado. Es decir, quien llega a llenar la vacante debe reconstruir y analizar en cuál etapa estaba la actividad para darle continuidad o en últimas empezar de nuevo, lo cual representa una pérdida de tiempo.

En este orden de ideas, y pensando en el mejoramiento continuo, se hace necesario replantear las estrategias de gestión del TH pues dicho proceso, tal y como se lleva a cabo en la actualidad, presenta serias deficiencias: el personal no estásuficientemente comprometido e involucrado con el SGC, en algunos casos no cuenta con las competencias necesarias, no está alineado con la cultura de calidad y no hay suficiente cohesión como para permitir unidad de propósito.

Frente a esta problemática, el objetivo de la presente investigación es proponer estrategias para el fortalecimiento del proceso de GTH en el SGC de la entidad. Para ello se realiza un análisis de la literatura existente, las normas de calidad y los testimonios de los involucrados en el proceso de GTH de Coralina.

Para lograr el objetivo planteado el presente trabajo se divide en cuatro secciones. En la primera se presenta la metodología empleada; en la segunda, los resultados del estudio con su respectivo diagnóstico, las estrategias sugeridas a la alta dirección de la entidad y la propuesta 
de plan de acción orientado a cumplir con las estrategias definidas previamente. La tercera sección está destinada al apartado de discusión y en la última se presentan las conclusiones del estudio.

\section{METODOLOGÍA}

La presente investigación asume un enfoque cualitativo y utiliza como estrategia metodológica el AC (análisis de contenido) según el derrotero ofrecido por Ruiz (2004). Se escogió el AC porque en los procesos de GTH, donde la comunicación tiene un lugar preponderante, dicha estrategia provee las herramientas para analizar satisfactoriamente tales procesos no solo a partir de la comunicación, sino del contexto en el cual se dan y el vínculo existente con el SGC de la entidad involucrada.

El AC ha sido poco utilizado en investigaciones relacionadas con los SGC, por lo que a continuación se presentan los pasos seguidos para la adopción de dicha técnica. El primer paso consistió en definir los elementos estructurales de la investigación a partir del AC (figura 1).

En el segundo paso se abordaron las estrategias de delimitación y determinación, según la técnica de AC, las cuales se definen en la tabla 1.

Figura 1. Elementos estructurales del AC

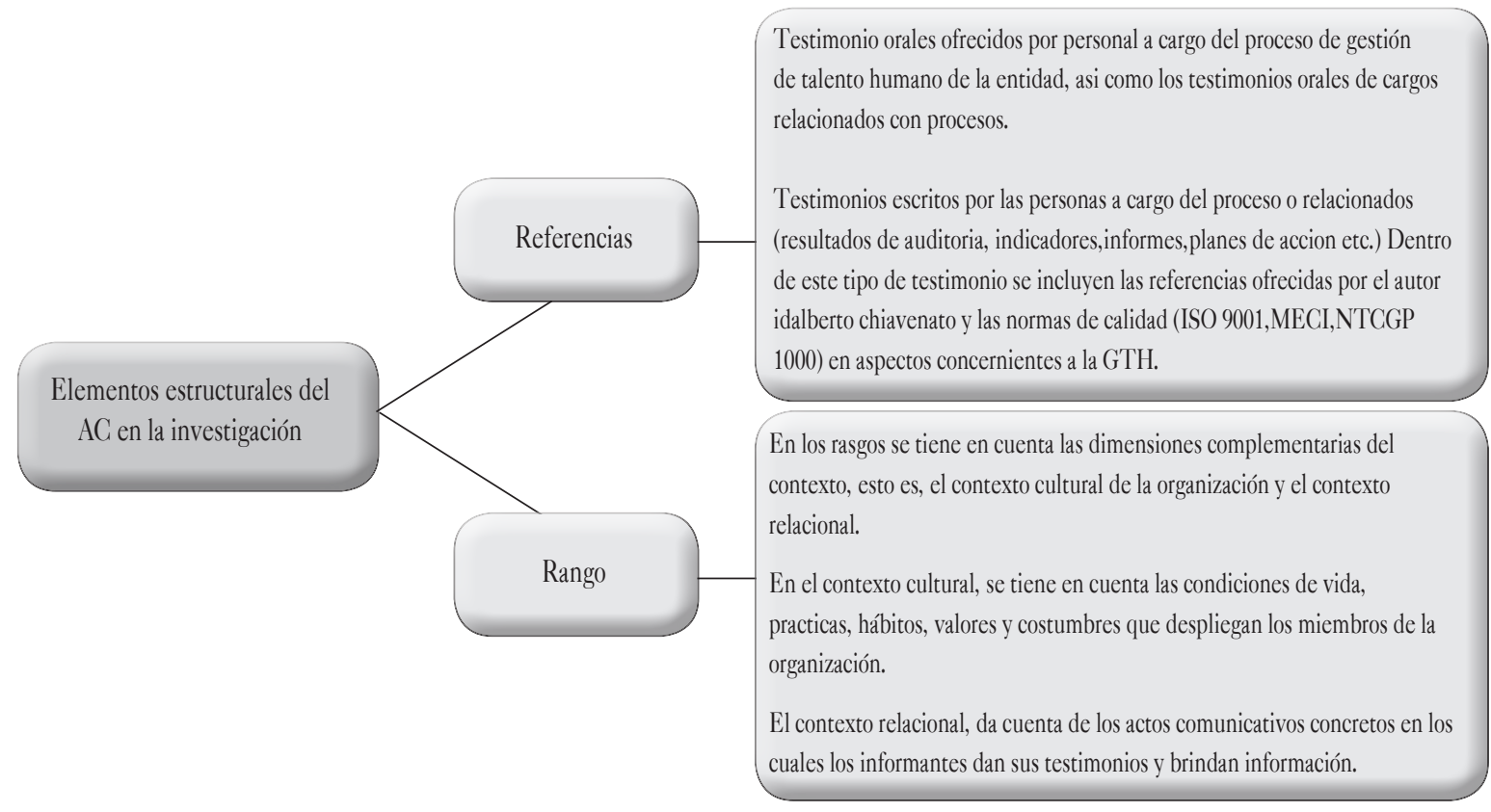

Fuente: elaboración propia a partir de Ruiz (2004). 
Leidy Marcela Romero Parra, Mireys Salcedo Lara

Tabla 1. Estrategias del estudio

\begin{tabular}{|c|c|c|}
\hline \multicolumn{3}{|c|}{ Estrategia de delimitación } \\
\hline \multicolumn{3}{|c|}{$\begin{array}{l}\text { Estrategia de delimitación intensiva } \\
\text { Integra al análisis todos los elementos presentes en los relatos de los informantes y testimonios escritos relacionados con el proceso de GTH y los } \\
\text { procesos para integrar, organizar, recompensar, desarrollar, retener y auditar al personal que labora para la entidad }\end{array}$} \\
\hline \multicolumn{3}{|c|}{ Estrategias de determinación } \\
\hline \multicolumn{3}{|c|}{$\begin{array}{l}\text { Estrategia intertextual } \\
\text { Utiliza el método discriminativo: los testimonios (orales y escritos) se organizan en categorías diferentes con el objeto de establecer comparaciones } \\
\text { entre ellos. Para eso se adopta el siguiente esquema: }\end{array}$} \\
\hline \multicolumn{3}{|c|}{ Categorías $(\mathrm{C})$ y subcategorías } \\
\hline $\begin{array}{l}\text { (C) } \text { Integración del TH } \\
\text { - } \quad \text { Reclutamiento } \\
\text { - } \quad \text { Selección } \\
\text { (C) } \text { Organización del TH } \\
\text { - } \quad \text { Diseño de puesto } \\
\text { - } \quad \text { Evaluación del desempeño } \\
\text { (C) Compensación del TH } \\
\text { - } \quad \text { Remuneraciones } \\
\text { - } \quad \text { Incentivos } \\
\text { - } \quad \text { Prestaciones }\end{array}$ & $\begin{array}{ll}\text { (C) } & \text { Desarrollo del TH } \\
\text { - } & \text { Formación } \\
\text { - } & \text { Desarrollo } \\
\text { - } & \text { Aprendizaje } \\
\text { - } & \text { Administración del co } \\
& \\
\text { (C) } & \text { Retención del TH } \\
\text { - } & \text { Higiene, seguridad } \\
\text { - } & \text { Relaciones laborales } \\
\text { - } & \text { Calidad de vida }\end{array}$ & $\begin{array}{l}\text { (C) Auditoría del TH } \\
\text { - } \quad \text { Bancos de datos } \\
\text { Sistemas de información administrativa }\end{array}$ \\
\hline $\begin{array}{l}\text { Organización } \\
\text { Testimonios orales ofrecidos por las personas } \\
\text { a cargo del proceso de GTH en la entidad y } \\
\text { por personas en cargos relacionados con el } \\
\text { proceso. } \\
\text { Testimonios escritos por las personas a cargo } \\
\text { del proceso o relacionadas (resultados de } \\
\text { auditorías, indicadores, informes, planes de } \\
\text { acción, etc.) }\end{array}$ & Comparativo & 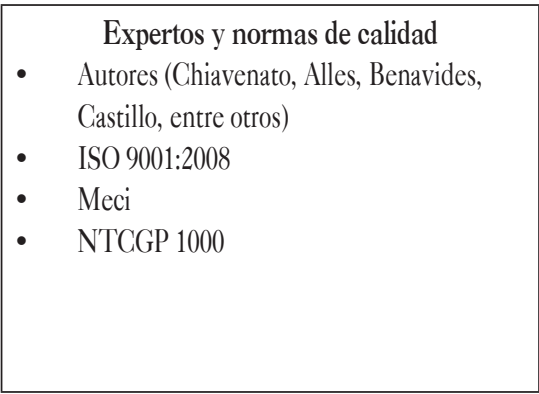 \\
\hline \multicolumn{3}{|c|}{$\begin{array}{l}\text { Estrategia extratextual } \\
\text { Contexto inmediato. Recopilación de testimonios teniendo en cuenta cambios en las normas de calidad. La ISO 9001:2015 trae importantes } \\
\text { cambios, como la introducción del concepto de gestión de cambio, el refuerzo del enfoque por procesos, la relevancia del liderazgo, requisitos más } \\
\text { explícitos y detallados para la comunicación, entre otros. Igualmente, se registran cambios en el Meci, que se actualizó en 2014, y modificaciones } \\
\text { en los subsistemas de control estratégico y de gestión. Este contexto es importante pues la entidad debería estar desarrollando planes para } \\
\text { adaptar su sistema a esos cambios. } \\
\text { Características especiales del informante. Aquí se tienen en cuenta los aspectos relacionados con la cultura organizacional y los rasgos predominantes } \\
\text { de las personas involucradas en el proceso de GTH que suministraron la información solicitada en el presente estudio }\end{array}$} \\
\hline
\end{tabular}

Fuente: elaboración propia a partir de Ruiz (2004). 
Figura 3. Esquema de triangulación

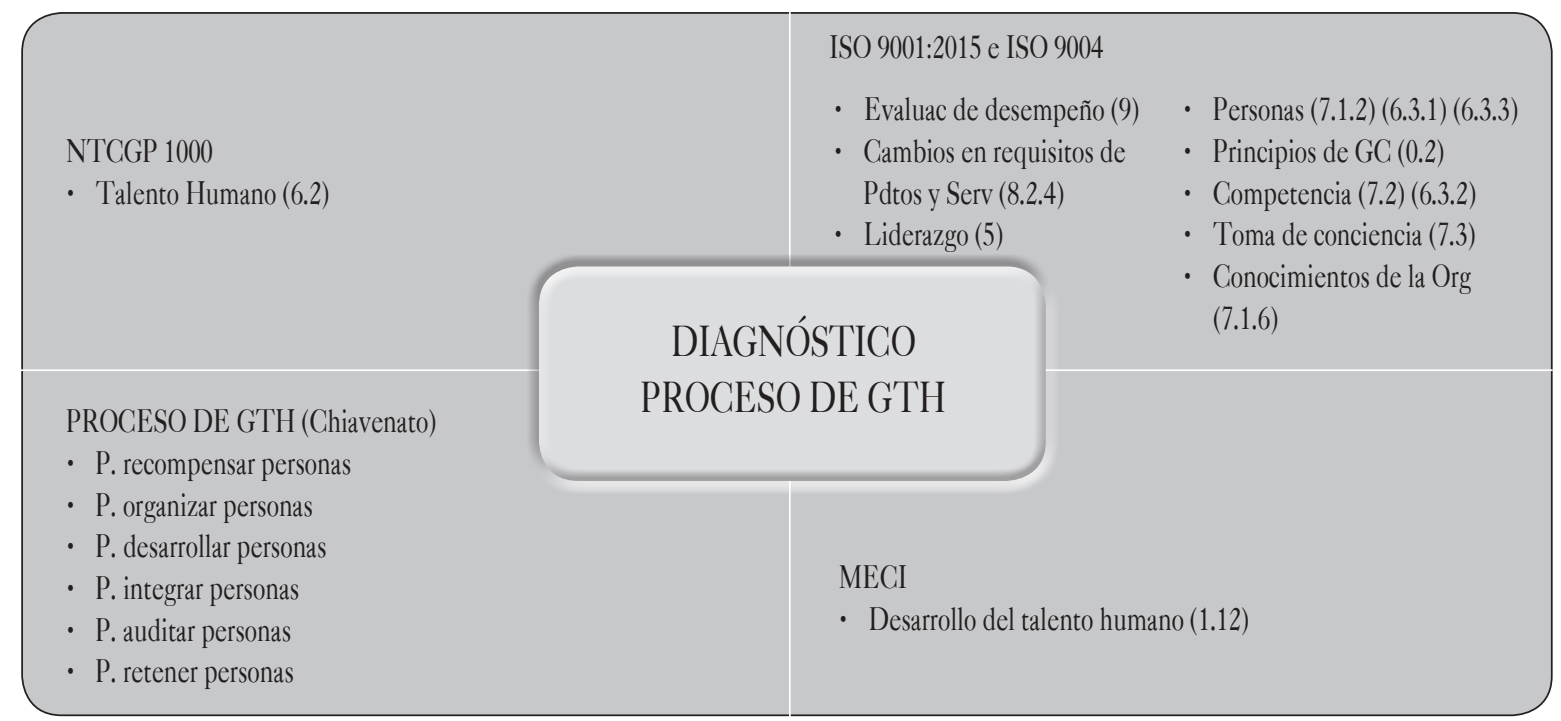

Fuente: elaboración propia.

\section{RESULTADOS Y DISCUSIÓN}

A continuación se hace una descripción sucinta de la entidad pública para seguidamente presentar el diagnóstico con los hallazgos más relevantes y a partir de allí proponer las estrategias y el plan de acción que debería ser implementado para articular el proceso de GTH con el SGC integrado de la entidad.

\section{Descripción de la entidad}

Creada en 1993, la entidad tiene como principal finalidad administrar el medio ambiente y los recursos naturales renovables, propendiendo al desarrollo sostenible del departamento Archipiélago de San Andrés, Providencia y Santa Catalina.

Como entidad pública orientada a la protección del medio ambiente, tiene unas funciones básicas que abarcan planeación; normatización; asesoría, coordinación y apoyo; administración; ejecución; investigación, educación e información en cuestiones medioambientales.
Maneja actualmente diecisiete proyectos, de los cuales catorce son propios, financiados por la entidad, y tres, en proceso de ejecución, son financiados por el FCA (Fondo de Compensación Ambiental). Adicionalmente, hay otros dos proyectos, pendientes de aprobación, que se están gestionando ante el Fonam (Fondo Nacional Ambiental).

El TH de la entidad se compone de 38 funcionarios, la mayoría profesionales, de los cuales $32 \%$ son funcionarios de carrera, $45 \%$ nombrados en provisionalidad y el restante $23 \%$, de libre nombramiento.

\section{Diagnóstico}

Teniendo en cuenta las categorías y subcategorías definidas previamente, el trabajo de campo realizado y las diferentes fuentes consultadas, a continuación se presenta el diagnóstico de la entidad utilizando para ello un cuadro de semaforización (tabla 2), según el nivel de avance observado en cada categoría. 
Tabla 2. Resultados del diagnóstico (semaforización)

\begin{tabular}{|c|c|c|c|c|}
\hline$\#$ & Componentes de la GTH & \multicolumn{2}{|c|}{ Situación actual } & $\mathrm{V}$ \\
\hline 1 & Procesos para integrar el TH & \multicolumn{2}{|c|}{$\begin{array}{l}\text { Hay procesos para integrar personal, pero no son idóneos para atraer personal más } \\
\text { competente }\end{array}$} & \\
\hline 2 & Procesos para organizar el TH & \multicolumn{2}{|c|}{$\begin{array}{l}\text { Existen procedimientos para evaluación del desempeño y diseño de puestos, pero se } \\
\text { cumplen parcialmente y no están bien formulados }\end{array}$} & \\
\hline 3 & $\begin{array}{l}\text { Procesos para recompensar } \\
\text { el TH }\end{array}$ & \multicolumn{2}{|c|}{$\begin{array}{l}\text { Si bien la remuneración y las prestaciones son definidas por el Gobierno, el programa de } \\
\text { incentivos, que depende de la entidad, tiene falencias y para la presente anualidad no } \\
\text { está establecido }\end{array}$} & \\
\hline 4 & Procesos para desarrollar el TH & \multicolumn{2}{|c|}{ No existe un verdadero proceso para desarrollar las habilidades personales } & \\
\hline 5 & Procesos para retener el TH & \multicolumn{2}{|c|}{ Son deficientes o inexistentes debido en gran parte a la falta de recursos } & \\
\hline 6 & Procesos para auditar el TH & \multicolumn{2}{|c|}{ Existen, pero presentan serias deficiencias } & \\
\hline 7 & $\begin{array}{l}\text { Procesos del SGC relacionados } \\
\text { con la GTH }\end{array}$ & \multicolumn{2}{|c|}{$\begin{array}{l}\text { El SGC está implementado y con procedimientos definidos, pero muchas de las } \\
\text { actividades de la GTH no se están llevando a cabo }\end{array}$} & \\
\hline$x$ & 2 & $28.6 \%$ & Inexistente & \\
\hline (1) & 5 & $71.4 \%$ & Parcial & \\
\hline (1) & 0 & $0 \%$ & Existe & \\
\hline
\end{tabular}

\begin{tabular}{|c|c|c|c|}
\hline \multicolumn{2}{|l|}{ 1. Procesos para integrar el TH } & \multicolumn{2}{|l|}{ 5. Procesos para retener el TH } \\
\hline Reclutamiento & (!) & Higiene y seguridad & $\times$ \\
\hline Selección & (!) & Relaciones laborales & $\mathbf{x}$ \\
\hline \multicolumn{2}{|l|}{ 2. Procesos para organizar el TH } & Calidad de vida & $\times$ \\
\hline Diseño de puestos & (!) & \multicolumn{2}{|l|}{ 6. Procesos para auditar el TH } \\
\hline Evaluación del desempeño & (1) & Banco de datos & (1) \\
\hline \multicolumn{2}{|l|}{ 3. Procesos para recompensar el TH } & Sistemas de información administrativa & (1) \\
\hline Remuneración & (1) & \multicolumn{2}{|l|}{ 7. Procesos SGC relacionados con la GTH } \\
\hline \multicolumn{2}{|l|}{ Incentivos } & \multirow[b]{2}{*}{ Objetivos de calidad de la GTH } & \multirow{2}{*}{ (1) } \\
\hline Prestaciones & ( & & \\
\hline \multicolumn{2}{|l|}{ 4. Procesos para desarrollar el TH } & \multirow[b]{2}{*}{ Realización de actividades planificadas en el área } & \multirow{2}{*}{ (1) } \\
\hline Formación & $x$ & & \\
\hline Desarrollo & $\mathrm{X}$ & Ambiente de trabajo & (1) \\
\hline Aprendizaje & 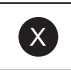 & Auditorías internas & (!) \\
\hline Administración del conocimiento & $\times$ & $\mathrm{PQR}$ & (!) \\
\hline
\end{tabular}


Leidy Marcela Romero Parra, Mireys Salcedo Lara

\begin{tabular}{|l|c|c|c|c|}
\hline \multicolumn{1}{|c|}{ Medidas } & N. & Existe & Parcial & Inexistente \\
\hline 1. Procesos para integrar el TH & 2 & 0 & 2 & 0 \\
\hline 2. Procesos para organizar el TH & 2 & 0 & 2 & 0 \\
\hline 3. Procesos para recompensar el TH & 3 & 2 & 0 & 1 \\
\hline 4. Procesos para desarrollar el TH & 4 & 0 & 0 & 4 \\
\hline 5. Procesos para retener el TH & 3 & 0 & 0 & 3 \\
\hline 6. Procesos para auditar el TH & 2 & 0 & 2 & 0 \\
\hline 7. Procesos SGC relacionados con la GTH & 5 & 0 & 5 & 0 \\
\hline Total medidas & 21 & 2 & 11 & 8 \\
\hline & & $9.5 \%$ & $\mathbf{5 2 . 4} \%$ & $38.1 \%$ \\
\hline
\end{tabular}

Fuente: elaboración propia.

Más de la mitad de los procesos de GTH y del SGC se llevan a cabo parcialmente o no son idóneos para el fin pretendido. Además, hay procesos que no reciben la importancia debida y que en la práctica no existen en la entidad, especialmente aquellos que tienen como finalidad desarrollar y retener al TH.

\section{Estrategias y planes de acción}

Para implementar las estrategias del área de TH y ejecutar los planes de acción se propone la creación de un
Comité Temporal para la Planeación del TH (figura 4) cuya duración dependerá del plazo que se tome la entidad para ejecutar el Plan de Acción, tras lo cual aquel se disolverá y dejará sus labores de control a cargo del área de TH.

Para la medición de la ejecución se propone adoptar el siguiente indicador, que arroja cifras porcentuales:

Ejecución del Plan de Acción=

\# de actividades ejecutadas \# de actividades programadas

Figura 4. Conformación Comité Temporal para la Planeación del TH

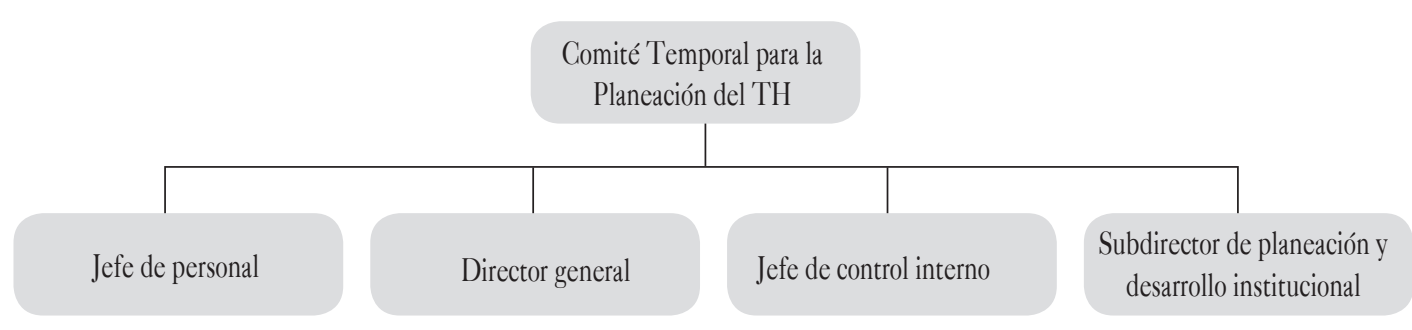

Fuente: elaboración propia.

En las tablas 3 a 15 se exponen de manera sucinta la estrategia y el respectivo plan de acción para cada subproceso de la GTH. 
Tabla 3. Estrategia y Plan de Acción, reclutamiento

\begin{tabular}{|l|l|}
\hline Estrategia & $\begin{array}{l}\text { Adopción de una estrategia de reclutamiento externo intensivo para ampliar el abanico de candidatos y aumentar la probabilidad } \\
\text { de escoger a los más idóneos }\end{array}$ \\
\hline Objetivo & Aumentar la base de candidatos a cargos de libre nombramiento y provisionales, así como el número de contratistas \\
\hline Responsable & Jefe del Área de TH \\
\hline \multicolumn{2}{|c|}{ Actividades del Plan de Acción } \\
\hline 1 & $\begin{array}{l}\text { Diseñar aviso de convocatoria con cargo, perfil, experiencia, salario y tipo de vinculación. Para los contratistas: objeto del contrato, } \\
\text { experiencia requerida, etc. }\end{array}$ \\
\hline 2 & Publicar aviso en medios impresos (revistas, diarios, etc.) \\
\hline 3 & Contactar asociaciones profesionales y universidades para que envíen candidatos a los cargos \\
\hline 4 & Colocar carteles o anuncios en lugares visibles y permitidos \\
\hline 5 & Animar al personal de la entidad a que envíe sus candidatos, si los tiene \\
\hline 6 & Publicar aviso de convocatoria en redes sociales \\
\hline 7 & Publicar aviso en página web utilizando la aplicación JS Jobs \\
\hline 8 & Anunciar convocatoria a través de radio y TV comunitarias \\
\hline 9 & Evaluar proceso de reclutamiento una vez concluido \\
\hline
\end{tabular}

Fuente: elaboración propia.

Tabla 4. Estrategia y Plan de Acción, selección

\begin{tabular}{|c|l|}
\hline Estrategia & $\begin{array}{l}\text { Adopción de una estrategia de selección de personal de libre nombramiento y provisional basada en el modelo de valor agregado, } \\
\text { orientado a seleccionar el TH que mayores competencias aporte a la entidad }\end{array}$ \\
\hline Objetivo & Incrementar el portafolio de competencias del TH \\
\hline Responsable & Jefe del Área de TH \\
\hline 1 & $\begin{array}{l}\text { Definir competencias (personales, del cargo, del área, esenciales y las demandadas por el SGC), requisitos de formación académica y } \\
\text { experiencia requerida por el cargo, según lo previsto en el Manual de Funciones de la entidad y lo requerido por el SGC }\end{array}$ \\
\hline 2 & Diseñar entrevistas; pruebas de conocimientos, sicológicas y de personalidad y técnicas de simulación, según requerimientos del cargo \\
\hline 3 & Programar fechas y horarios para la aplicación de entrevistas y pruebas \\
\hline 4 & Aplicar entrevistas y pruebas \\
\hline 5 & Evaluar las pruebas \\
\hline 6 & Evaluar competencias de los entrevistados utilizando el formato Evaluación de Competencias \\
\hline 7 & $\begin{array}{l}\text { Identificar candidatos que además de cumplir con las competencias exigidas para desempeñar el cargo posean competencias que } \\
\text { agreguen valor a la entidad }\end{array}$ \\
\hline 8 & $\begin{array}{l}\text { Elegir al candidato que reuniendo las competencias requeridas posea la mayor cantidad de competencias agregadas que resulten valiosas } \\
\text { para la entidad }\end{array}$ \\
\hline 9 & Realizar exámenes médicos de ingreso \\
\hline 10 & Agradecer a los participantes en el proceso de selección e informar acerca de su finalización \\
\hline
\end{tabular}

Fuente: elaboración propia. 
Tabla 5. Estrategia y Plan de Acción, diseño de puestos

\begin{tabular}{|c|l|}
\hline Estrategia & $\begin{array}{l}\text { Adopción en la entidad de una estrategia para rediseño de puestos basada en el modelo de las contingencias, que tiene en cuenta la } \\
\text { naturaleza cambiante del entorno, la tecnología y las personas }\end{array}$ \\
\hline Objetivo & Lograr una mayor flexibilidad de la entidad adaptando el diseño de puestos a la naturaleza cambiante del entorno \\
\hline Responsable & Jefe del Área de TH \\
\hline & \multicolumn{1}{|c|}{ Actividades del Plan de Acción } \\
\hline 1 & $\begin{array}{l}\text { Investigar nuevos adelantos y desarrollos que guarden una relación directa con las tareas que demanda el puesto y las competencias } \\
\text { requeridas }\end{array}$ \\
\hline 2 & Rediseñar el contenido del puesto (tareas o atribuciones) teniendo en cuenta los nuevos adelantos \\
\hline 3 & Definir responsabilidad del puesto (a quién debe reportar) \\
\hline 4 & Definir autoridad del puesto (a quién debe dirigir o supervisar) \\
\hline 5 & Rediseñar el puesto de trabajo teniendo en cuenta: \\
\hline 5.1 & Variedad de la tarea, equipamientos, actividades, habilidades y potencial innovador \\
\hline 5.2 & Autonomía del puesto \\
\hline 5.3 & Significado de las tareas (conocimiento profundo de las implicaciones, interdependencias y consecuencias del trabajo) \\
\hline 5.4 & Identidad con las tareas (identificación con el trabajo, pertenencia) \\
\hline 5.5 & $\begin{array}{l}\text { Realimentación (información de retorno o retroalimentación que recibe la persona para administrar los resultados de lo que hace y } \\
\text { generar una autoevaluación) }\end{array}$ \\
\hline 6 & Establecer secuencia gradual para la asignación futura de nuevas responsabilidades a fin de enriquecer el puesto \\
\hline
\end{tabular}

Fuente: elaboración propia.

Tabla 6. Estrategia y Plan de Acción, EDL (evaluación del desempeño laboral)

\begin{tabular}{|l|l|}
\hline Estrategia & $\begin{array}{l}\text { Articular el proceso de EDL con las competencias para el cargo, definidas previamente en los procesos de selección y el Manual } \\
\text { de Funciones involucrando a todos los funcionarios independientemente del tipo de vinculación }\end{array}$ \\
\hline Objetivo & Democratizar la medición del desempeño laboral con énfasis en las competencias requeridas por el cargo \\
\hline Responsable & Jefe del Área de TH \\
\hline \\
\hline 1 & Incluir en las EDL a funcionarios provisionales y de libre nombramiento \\
\hline 2 & $\begin{array}{l}\text { Sumar a las competencias del Manual de Funciones del cargo las competencias definidas para el proceso de selección en el formato de } \\
\text { Evaluación de Competencias }\end{array}$ \\
\hline 3 & Diseñar procedimiento para evaluar con igual periodicidad a los demás funcionarios independientemente del tipo de vinculación \\
\hline
\end{tabular}

Fuente: elaboración propia. 
Tabla 7. Estrategia y Plan de Acción, incentivos

\begin{tabular}{|c|c|c|}
\hline \multicolumn{2}{|c|}{ Estrategia } & $\begin{array}{l}\text { Adopción del Plan de Incentivos, paralelo al de funcionarios de carrera, para estimular a los demás funcionarios que tengan un } \\
\text { desempeño laboral sobresaliente }\end{array}$ \\
\hline \multicolumn{2}{|c|}{ Objetivo } & Democratizar los incentivos \\
\hline \multicolumn{2}{|c|}{ Responsable } & Jefe del Área de TH \\
\hline \multicolumn{3}{|r|}{ Actividades del Plan de Acción } \\
\hline 1 & \multicolumn{2}{|c|}{ Establecer presupuesto para el Plan de Incentivos 2017} \\
\hline 2 & \multicolumn{2}{|r|}{ Diseñar Plan General de Incentivos 2017 de modo que incluya a todos los funcionarios de la entidad } \\
\hline 3 & \multicolumn{2}{|c|}{ Analizar resultados globales de la EDL } \\
\hline 4 & \multicolumn{2}{|c|}{ Aplicar incentivos de ley a funcionarios de carrera } \\
\hline 5 & \multicolumn{2}{|r|}{ Aplicar incentivos a los mejores desempeños de funcionarios que no son de carrera } \\
\hline
\end{tabular}

Fuente: elaboración propia.

Tabla 8. Estrategia y Plan de Acción, formación, desarrollo y aprendizaje

\begin{tabular}{|c|l|}
\hline Estrategia & Adopción del Plan de Capacitaciones, basado en la formación por competencias para todo el personal \\
\hline Objetivo & $\begin{array}{l}\text { Enfocar el subproceso de formación, desarrollo y aprendizaje en competencias valiosas para la entidad, orientadas a mejorar el } \\
\text { desempeño de la planta de cargos, fomentar el crecimiento personal y adquirir nuevas habilidades y destrezas }\end{array}$ \\
\hline Responsable & Jefe del Área de TH \\
\hline \multicolumn{2}{|c|}{ Actividades del Plan de Acción } \\
\hline 1 & $\begin{array}{l}\text { Identificar necesidades en cuanto al desarrollo de las competencias personales, del cargo, del área; las esenciales para la entidad } \\
\text { (relacionadas con la estrategia, la misión, los valores, la cultura organizacional, etc.) y las que demanda el SGC }\end{array}$ \\
\hline 2 & Formulación de las capacitaciones (individuales, por área o globales) requeridas \\
\hline 3 & $\begin{array}{l}\text { Definición de la(s) técnica(s) que se empleará(n) en cada una de las capacitaciones (lectura, película, instrucción programada, en clase, } \\
\text { por computador, e-learning, tipo coaching, etc.) }\end{array}$ \\
\hline 4 & Definición de los ambientes para las capacitaciones (físicos o virtuales) \\
\hline 5 & Ejecución del Plan de Capacitaciones \\
\hline
\end{tabular}

Fuente: elaboración propia.

Tabla 9. Estrategia y Plan de Acción, administración del conocimiento

\begin{tabular}{|l|l|}
\hline Estrategia & Creación del Centro de Aprendizaje de la entidad \\
\hline Objetivo & Crear dentro de la entidad el Centro de Aprendizaje, enfocado en la administración y difusión del conocimiento \\
\hline Responsable & Director general \\
\hline \multicolumn{2}{|c|}{ Actividades del Plan de Acción } \\
\hline 1 & Definir dotación requerida por el Centro de Aprendizaje \\
\hline 2 & Determinar localización idónea \\
\hline 3 & Definir presupuesto \\
\hline 4 & Sumar apoyos (locales, nacionales e internacionales) para la consecución de los recursos requeridos para la creación del Centro \\
\hline 5 & Ejecutar el proyecto \\
\hline 6 & Establecer convenios con otras entidades de orden local, nacional e internacional para intercambio de conocimiento \\
\hline
\end{tabular}

Fuente: elaboración propia. 
Leidy Marcela Romero Parra, Mireys Salcedo Lara

Tabla 10. Estrategia y Plan de Acción, higiene y seguridad

\begin{tabular}{|l|l|}
\hline Estrategia & Implementación del Sistema de Gestión de la Seguridad y Salud en el Trabajo (SG-SST) \\
\hline Objetivo & Propiciar condiciones de higiene y seguridad a fin de anticipar, reconocer, evaluar y controlar los riesgos que puedan afectar la SST \\
\hline Responsable & Director general \\
\hline \multicolumn{2}{|c|}{ Actividades del Plan de Acción } \\
\hline 1 & Contratar un profesional en SST \\
\hline 2 & Realizar evaluación inicial \\
\hline 3 & Diseñar plan de mejora en SST según los resultados de la evaluación inicial \\
\hline 4 & Ejecutar actividades planeadas \\
\hline 5 & Hacer seguimiento y establecer mejoras según los resultados del seguimiento \\
\hline 6 & Solicitar visita de la ARL para auditar SG-SST. \\
\hline
\end{tabular}

Fuente: elaboración propia.

Tabla 11. Estrategia y Plan de Acción, relaciones laborales

\begin{tabular}{|l|l|}
\hline Estrategia & Adopción del PAE (Programa de Ayuda a Empleados) \\
\hline Objetivo & Resolver los conflictos y problemas con el TH generados en la entidad \\
\hline Plazo & Asignado por la entidad \\
\hline Responsable & Director general \\
\hline Actividades del Plan de Acción \\
\hline 1 & Conformar el Comité PAE \\
\hline 2 & Establecer periodicidad de las reuniones del Comité \\
\hline 3 & Difundir la información relacionada con el Comité PAE (funciones, miembros, trámite de las quejas) \\
\hline
\end{tabular}

Fuente: elaboración propia.

Tabla 12. Estrategia y Plan de Acción, acoso laboral

\begin{tabular}{|l|l|}
\hline Estrategia & Creación del CCL (Comité de Convivencia Laboral) \\
\hline Objetivo & Resolver los casos de acoso laboral de la entidad \\
\hline Responsable & Director general \\
\hline \multicolumn{1}{|c|}{ Actividades del Plan de Acción } \\
\hline 1 & Elección de los dos representantes de la Alta Dirección, con sus respectivos suplentes, por parte del director general \\
\hline 2 & Elección de los dos representantes de los funcionarios con sus respectivos suplentes, por medio de votación \\
\hline 3 & Elección por parte de los miembros del CCL de presidente(a) y secretario(a) \\
\hline 4 & $\begin{array}{l}\text { Solicitar a la ARL capacitación al CCL en acoso laboral, trámite de quejas de acoso, liderazgo, comunicación asertiva, resolución de } \\
\text { conflictos, diligenciamiento de las actas de reuniones y elaboración de los informes que se presentan a la Alta Dirección }\end{array}$ \\
\hline
\end{tabular}

Fuente: elaboración propia. 
Tabla 13. Estrategia y Plan de Acción, calidad de vida

\begin{tabular}{|l|l|}
\hline Estrategia & Adopción Programa de Bienestar \\
\hline Objetivo & Diseñar Programa de Bienestar para el TH de la entidad \\
\hline Responsable & Director general \\
\hline \multicolumn{2}{|c|}{ Actividades del Plan de Acción } \\
\hline 1 & Conformación del equipo de trabajo que diseñará el programa. \\
\hline 2 & Diseño del programa según los objetivos básicos planteados en la estrategia \\
\hline 3 & Asignación del presupuesto para las actividades del programa. \\
\hline 4 & Ejecución del programa. \\
\hline
\end{tabular}

Fuente: elaboración propia.

Tabla 14. Estrategia y Plan de Acción, banco de datos

\begin{tabular}{|c|l|}
\hline Estrategia & Manejo interconectado y sistematizado de bases de datos relacionadas con el personal \\
\hline Objetivo & Sistematizar las bases de datos del personal y crear aquellas que hagan falta \\
\hline Responsable & Jefe del Área de TH \\
\hline \multicolumn{2}{|c|}{ Actividades del Plan de Acción } \\
\hline 1 & Crear formato para consentimiento informado \\
\hline 2 & Imprimirlo y hacerlo firmar de los funcionarios \\
\hline 3 & Crear registros necesarios \\
\hline 4 & Crear macros en Excel con los datos no sistematizados \\
\hline 5 & Actualizar información cada que ocurran cambios \\
\hline
\end{tabular}

Fuente: elaboración propia.

Tabla 15. Estrategia y Plan de Acción, sistemas de información administrativa

\begin{tabular}{|c|l|}
\hline Estrategia & Diseño de base de datos por funcionario, con información relevante \\
\hline Objetivo & Filtrar bases de datos del personal a fin de disponer de información relevante para la toma de decisiones administrativas \\
\hline Responsable & Jefe del Área de TH \\
\hline \multicolumn{2}{|c|}{ Actividades del Plan de Acción } \\
\hline 1 & $\begin{array}{l}\text { Definir los criterios de la plantilla que se va a crear con los datos relevantes que va a contener (salario, fecha de ingreso, vacaciones, } \\
\text { puesto, experiencia, habilidades y conocimientos, entrenamiento recibido, desempeño, entre otros) }\end{array}$ \\
\hline 2 & Definir los gráficos que contendrá la plantilla (de barras, circulares, de dispersión, etc.) \\
\hline 3 & $\begin{array}{l}\text { Crear plantilla con información relevante de cada empleado, especialmente la que sea de uso frecuente por parte de la administración } \\
\text { en el proceso de toma de decisiones }\end{array}$ \\
\hline 4 & Actualizar periódicamente la información \\
\hline
\end{tabular}

Fuente: elaboración propia. 
Las estrategias y los planes de acción planteados para cada una de las categorías de la GTH deberían ser suficientes para mejorar este proceso y lograr su articulación con el SGC de la entidad y muy especialmente con la norma de calidad ISO 9004:2009, que enfatiza la necesidad de gestionar bien el TH (tabla 16), la cual fue tenida en cuenta para formular la propuesta.

Los resultados obtenidos concuerdan con los planteamientos de diferentes investigadores (Jericó, 2001; Castillo, 2006; Carballo, 2006; Jiménez, 2007; Chiavenato, 2009; Hernández, Salazar, Rodríguez y Fleitas, 2010; Medina, Delgado y Lavado, 2012; Alles, 2016) y con las mismas normas de calidad, como las ISO 9004 y 9001 en su versión de 2015 (Álvarez, 2017), que exponen la necesidad de las organizaciones no solo de medir a través de indicadores la GTH (Manrique, Ferrer, Muñoz y Martínez, 2010; Cuesta y Valencia, 2014), sino también de formar a su TH por competencias. Claro está, dichas competencias ya no pueden estar supeditadas al cargo que se desempeña, esto es, hay algunas que aunque no están esbozadas en los manuales de funciones son necesarias para alcanzar la excelencia. Por ejemplo, las relacionadas con el SGC y las que son o serán esenciales para la organización. Más aún, no es suficiente adquirir dichas competencias para lograr la excelencia pues el conocimiento debe además ser gestionado, de tal manera que se cree una verdadera riqueza en capital intelectual.

Los resultados del diagnóstico efectuado muestran que, por sí sola, la implementación de un SGC es insuficiente para mejorar las competencias y condiciones del TH, lo cual contradice los resultados de las investigaciones efectuadas por Méndez y Fandiño (2010) y Estacio Maigual (2012) pues otras condiciones, como la cultura organizacional (Salcedo y Romero, 2006; Broncano y Rubio, 2009; Montealegre, 2015) o el clima laboral (Zárraga y Bonache, 2005; Bordas, 2016), también juegan un importante rol.

Tabla 16. Esquema ISO 9004:2009 con respecto a la GTH incluido en las estrategias propuestas

\begin{tabular}{|l|l|l|}
\hline \multicolumn{1}{|c|}{ Gestión de las personas (6.3.1) } & Competencia de las personas (6.3.2) & Participación y motivación de las personas (6.3.3) \\
\hline Ambientes de trabajo orientados a fomentar: & Para identificar, desarrollar y mejorar & Aumentar la motivación y participación de las \\
- Crecimiento personal & las competencias de las personas, la & personas mediante actividades tales como \\
- Aprendizaje & organización debe: & - Desarrollar procesos para compartir conocimiento \\
- Transferencia de conocimientos & -Identificar competencias requeridas a & - Introducir un sistema de reconocimiento y \\
- Trabajo en equipo & corto y largo plazo & recompensa adecuado \\
\cline { 1 - 1 } $\begin{array}{l}\text { Procesos que faculten a las personas para } \\
\text { - Traducir objetivos de la organización a } \\
\text { objetivos individuales }\end{array}$ & - Identificar competencias disponibles & - Establecer un sistema de calificación de \\
- Identificar limitaciones al desempeño & - Implementar acciones para mejorar o & desarrollo de las personas \\
- Asumir la responsabilidad en resolución de & - Revisar y evaluar la eficacia de las & - Revisar constantemente los niveles de satisfacción \\
problemas & acciones adelantadas & - Proporcionar oportunidades para realizar tutorías y \\
- Evaluar desempeño & adiestramiento profesional (coaching) \\
- Buscar oportunidades para aumentar & & \\
competencias y experiencia & & \\
- Promover trabajo en equipo y sinergias & & \\
- Compartir información, conocimiento y & & \\
experiencia & & \\
\hline
\end{tabular}

Fuente: elaboración propia a partir de la norma ISO 9004:2009. 


\section{CONCLUSIONES}

Los resultados obtenidos en la fase de diagnóstico muestran que, si bien la entidad ha cumplido en cierta medida con los requisitos del SGC, es necesario profundizar en su implementación pues las auditorías hechas al sistema han arrojado no conformidades consecuencia directa de la falta de preparación y motivación del personal. De hecho, el área de TH ni siquiera ha controlado el cumplimiento de los objetivos del SGC en lo que le corresponde.

Dentro de los hallazgos del diagnóstico se encuentra que por tratarse de una entidad pública la remuneración y las prestaciones son fijadas por el Gobierno nacional, lo que puede ser un impedimento para atraer personal idóneo. Sin embargo, más que la remuneración y las prestaciones, lo que se advierte es que una de las principales problemáticas que aqueja a la entidad es la falta de recursos para ejecutar programas en pro del TH, además de cierta falta de compromiso y motivación pues las actividades del SGC se hacen más para cumplir y pasar las auditorías que por una férrea convicción en las bondades del sistema. Esta situación seguramente no es muy diferente de la que se presenta en otras entidades públicas, donde la implementación de los SGC ha sido una imposición gubernamental sin una labor previa orientada a que los funcionarios interioricen los principios de la cultura de la calidad.

La situación arriba planteada implica la existencia de un SGC desactualizado y con muchas de las actividades que se debían ejecutar en 2017 sin programar. De ahí que, según algunos de los testimonios ofrecidos, la percepción de bienestar del TH ha declinado, lo que va en contravía del mejoramiento continuo, que es la esencia de cualquier SGC.

Dentro de la entidad el TH tampoco tiene el peso que debería tener. Eso ha menguado la efectividad del SGC pues, como se había anotado antes, las personas son fundamentales para lograr el éxito de los sistemas de gestión y conseguir su participación se ha convertido en uno de los principales retos que enfrenta actualmente la alta dirección de la entidad, máxime cuando resulta evidente que los procesos de formación por competencias no se ejecutan.

Existe cierta discriminación entre funcionarios en cuanto a capacitaciones pues, según algunas declaraciones, los funcionarios en provisionalidad no tienen derecho a aquellas. Igualmente, se encontró que las evaluaciones de desempeño que permiten a los funcionarios acceder a ciertos beneficios e incentivos solo son realizadas a los funcionarios de carrera.

De los resultados arrojados por este estudio se concluye que en todas las etapas del proceso de GTH se presentan fallas que afectan la articulación entre TH y SGC, necesaria para lograr un proceso exitoso y sostenido, según lo previsto en las normas de calidad y muy especialmente en la NTC-ISO 9004:2009.

Finalmente, en apoyo a lo dicho hasta aquí, los resultados del diagnóstico muestran que actualmente más de la mitad de los procesos relacionados con la GTH y el SGC se llevan a cabo parcialmente o no son idóneos para el fin pretendido. A esto se suman aquellos procesos que no reciben la importancia debida o que sencillamente no existen en la entidad, como los que tienen por finalidad desarrollar y retener al $\mathrm{TH}$. 


\section{REFERENCIAS}

Alles, M. (2006). Desarrollo del talento humano basado en competencias. Buenos Aires: Granica.

Alles, M. (2016). Selección por competencias. Buenos Aires: Granica.

Álvarez, S. (2017). Plan de acción para perfeccionar el Sistema de Calidad del Centro Nacional Coordinador de Ensayos Clínicos de Cuba. Infodir, 24, 4-12.

Atehortúa, F., Bustamante, R. E. y Valencia, J. A. (2008). Sistema de gestión integral. Una sola gestión, un solo equipo. Medellín: Universidad de Antioquia.

Benavides, C. y Quintana, C. (2015). Gestión del conocimiento y calidad total. Madrid: Díaz de Santos.

Bordas, M. J. (2016). Gestión estratégica del clima laboral. Madrid: Uned.

Broncano, S. y Rubio, M. (2009). El factor humano en los sistemas de gestión de calidad del servicio: un cambio de cultura en las empresas turísticas. Cuadernos de Turismo, 23, 129-147.

Carballo, R. (2006). Innovación y gestión del conocimiento: modelo, metodología, sistemas y herramientas de innovación. Madrid: Díaz de Santos.

Castillo, J. (2006). Administración de personal. Un enfoque hacia la calidad. Bogotá: Ecoe.

Chiavenato, I. (2009). Gestión del talento humano. México: McGraw-Hill.

Cuesta, A. y Valencia, M. (2014). Indicadores de gestión humana y del conocimiento en la empresa. Bogotá: Ecoe.
Estacio Maigual, S. L. (2012). Efectos de la implementación del sistema de gestión de la calidad bajo el modelo NTC-ISO 9001 en la gestión del recurso humano. Signos, 4(1), 35-43. Doi: https://doi. org/10.15332/s2145-1389.2012.0001.02

Gutiérrez, S. y Rubio, M. (2009). El factor humano en los sistemas de gestión del servicio: un cambio de cultura en las empresas turísticas. Cuadernos de Turismo, 23, 129-147.

Hamel, G. y Breen, B. (2012). El futuro de la administración. Bogotá: Norma.

Hernández, I., Salazar, D., Rodríguez, M. y Fleitas, M. S. (2010). Estrategias para integrar la gestión del capital humano a la gestión empresarial. Ingeniería Industrial, 31(3), 1-10.

Icontec (Instituto Colombiano de Normalización y Certificación) (2005). Sistemas de gestión de la calidad: fundamentos y vocabulario. NTC ISO 9000. Bogotá: Icontec.

Jericó, P. (2001). Gestión del talento. Madrid: Prentice Hall.

Jiménez, D. (2007). Manual de recursos humanos. Madrid: Esic.

Manrique, M., Ferrer, J., Muñoz, C. y Martínez, M. (2010). El Balanced Scorecard en el área de recursos humanos: caso Gerencia de Compensaciones de una empresa de alimentos. Cuadernos de Estudios Empresariales, 20, 145-176.

Medina, A. F., Delgado, A. M. y Lavado, P. A. (2012). Fundamentos de un sistema de gestión humana por competencias para soportar la estrategia organizacional en una pyme del sector de la industria de las 
artes gráficas en Cali (Colombia). Estudios Gerenciales, 28(122), 121-138. Doi: https://doi.org/10.1016/ S0123-5923(12)70197-3

Méndez, S. E. y Fandiño, R. (2010). Efectos de la implementación del sistema de gestión de calidad en la gestión humana del Centro de Gestión Administrativa del Sena. Signos, 2(1), 15-28. Doi: https://doi. org/10.15332/s2145-1389.2010.0001.01

Montealegre, J. V. (2015). La cultura organizacional y la resistencia al cambio. Madrid: Editorial Académica Española.

Pereda, S. y Berrocal, F. (2011). Dirección y gestión de recursos humanos por competencias. Madrid: Centro de Estudios Ramón Areces.

Petrick, J.y Furr, D. (2003).La calidad total en la dirección de recursos humanos. Barcelona: Gestión 2000.
Ruiz, A. (2004). Texto, testimonio y metatexto: el análisis de contenido en la investigación en educación. En A. Jiménez y A. Torres (comps.), $L a$ práctica investigativa en ciencias sociales (pp. 45-62). Bogotá: UPN.

Salcedo, I. y Romero, J. J. (2006). Cultura organizacional y gestión de la calidad en una empresa del Estado venezolano. Revista Venezolana de Gerencia (RVG), 11(33), 83-104.

Sánchez, J. R. (2011). La empresa humana. Las organizaciones empresariales y el hombre. Tomo I. Madrid: Visión.

Zárraga, C. y Bonache, J. (2005). Equipos de trabajo para la gestión del conocimiento: la importancia de un clima adecuado. Cuadernos de Economía y Dirección de la Empresa, 22, 27-48. 